\title{
A Two-stage Game-theoretic Method for Residential PV Panels Planning Considering Energy Sharing Mechanism
}

\author{
$\mathrm{Xu} \mathrm{Xu}$, Member, IEEE, Jiayong Li, Member, IEEE, Yan Xu, Senior Member, IEEE, \\ Zhao Xu, Senior Member, IEEE, Chun Sing Lai, Member, IEEE
}

\begin{abstract}
This paper proposes a novel two-stage game-theoretic residential photovoltaic (PV) panels planning framework for distribution grids with potential PV prosumers. One innovative contribution is that a residential PV panels location-allocation model is integrated with the energy sharing mechanism to increase economic benefits to PV prosumers and meanwhile facilitate the reasonable installation of residential PV panels. The optimization of residential PV panels planning decisions is formulated as a two-stage model. In the first stage, we develop a Stackelberg game based stochastic bi-level energy sharing model to determine the optimal sizing of $\mathrm{PV}$ panels with uncertain PV energy output, load demand, and electricity price. Instead of directly solving the proposed bi-level energy sharing problem by using commercial solvers, we develop an efficient descend search algorithm-based solution method which can significantly improve the computation efficiency. In the second stage, we propose a stochastic programming based residential PV panels deployment model for all PV prosumers. This model is formulated as an optimal power flow (OPF) problem to minimize active power loss. Finally, simulations on an IEEE 33-node and 123-node test systems demonstrate the effectiveness of the proposed method.
\end{abstract}

Index Terms - Residential photovoltaic panels planning, energy sharing mechanism, Stackelberg game, descend search algorithm, optimal power flow problem

\section{NOMENCLATURE}

Indices and sets

Index of nodes/lines/agents

This work is supported by the National Natural Science Foundation of China (Grant No. 71971183). This work is also supported by Hong Kong RGC Theme Based Research Scheme Grants No. T23-701/14N. The work of J. Li is supported by the National Natural Science Foundation of China (Grant No. 51907056). Y. Xu's work is supported by Nanyang Assistant Professorship from Nanyang Technological University, Singapore.

$\mathrm{X}$. Xu is with the Department of Electrical Engineering, The Hong Kong Polytechnic University, Hung Hom, Hong Kong and also with the School of Electrical and Electronic Engineering, Nanyang Technological University, Singapore. (email: benxx.xu@connect.polyu.hk).

$\mathrm{J}$. Li is with the College of Electrical and Information Engineering, Hunan University, Changsha, China. (email: jyli@hnu.edu.cn).

$\mathrm{Y}$. Xu is with the School of Electrical and Electronic Engineering, Nanyang Technological University, Singapore. (email: xuyan@ntu.edu.sg).

Z. Xu is with both Shenzhen Research Institute and Department of Electrical Engineering, The Hong Kong Polytechnic University, Hung Hom, Hong Kong. (email: eezhaoxu@polyu.edu.hk).

C. S. Lai is with the Department of Electronic and Computer Engineering, Brunel University London, London, UB8 3PH, UK and also with the Department of Electrical Engineering, Guangdong University of Technology, Guangzhou, China 510006 (email: chunsing.lai @brunel.ac.uk).
$k / i$
$w$
$\mathcal{N} / \mathcal{L} / \mathcal{T} / \mathcal{W}$
$\mathcal{C}_{j}$
$\mathcal{N}^{P V} / \mathcal{N}^{C}$
Index of prosumer agents/consumer agents
Index of time periods
Index of scenarios

\section{Set of child nodes of node $j$}
Set of nodes/lines/time periods/scenarios
Set of prosumer agents/consumer agents

Variables

$P_{j t w} / Q_{j t w}$

$v_{j t w}$

$l_{j t w}$

$p_{j t w} / q_{j t w}$

$P_{i t w}^{P V C}$

$P_{k t w}^{P V g}$

$P_{\text {ktw }}^{\text {Curtail }}$

$P_{j t w}^{G}$

$c_{t w}^{U}$

$u_{k}^{P V}$

$\phi^{P V}$

$\lambda_{\text {itw }}^{L}$

$\mu_{i t w}^{P V C} / \mu_{i t w}^{G}$

$U_{i t w}^{C}$

$\operatorname{Rev}^{C}$

Parameters

$c_{t w}^{G} / c_{t w}^{M C P}$

$c_{i n v}^{P V} / c_{o \& m}^{P V}$

$c^{E}$

$r^{C}$

$w_{i}^{E}$

$c_{i t w}^{W T P}$

$p_{w}$

$\gamma_{t w}^{P V} / \gamma_{t w}^{L}$

$P_{j t w}^{L} / Q_{j t w}^{L}$

$E_{\text {size }}^{P V}$

$E^{P V \max }$

Active/reactive power flow on distribution line $j$ at time $t$ in scenario $w$

Squared voltage magnitude on node $j$ at $t, w$

Squared line current magnitude on line $j$ at $t, w$

Active/reactive power injection on node $j$ at $j, t, w$

PV electricity sold to the consumer agent $i$ at $t, w$

PV electricity sold by the prosumer agent $k$ at $t, w$

PV energy curtailment on prosumer agent node $k$ at $t, w$

Electricity purchased from the utility grid by agent $j$ at $t, w$

Uniform price applied to all consumer agents at $t, w$

Number of PV panels purchased by prosumer agent $k$

Total installation number of PV panels

Dual variable of supply-demand balance $(6 \mathrm{~g})$

Dual variable of inequality constraint $(6 \mathrm{~h}) /(6 \mathrm{i})$

The utility function of consumer agent $i$ at $t, w$

Revenue of the coalition formed by all prosumer agents

Electricity price /wholesale market clearing price at $t, w$

Investment cost/operation \& maintenance cost of each PV panel

Greenhouse gas emission cost

The proportion of the incomes of sharing PV electricity to consumer agents via the energy sharing platform

Individual weight factor for greenhouse gas emissions reduction of consumer agent $i$

Willingness-to-pay of consumer agent $i$ at $t, w$

Probability of uncertainty scenario $w$

PV energy output factor/load factor at $t, w$

Active/reactive load on node $j$ at $t, w$

Size of each PV panel

PV hosting capacity of the distribution grid

$u_{k}^{P V \max } \quad$ Maximum allowable installation number of $\mathrm{PV}$ panels on 
node prosumer agent node $k$

$v^{\min } / v^{\max }$ Squared lower/upper bound of voltage magnitude

$l_{j}^{\max } \quad$ Squared upper bound of current magnitude on distribution line $j$

$\eta^{P V} \quad$ Efficiency of PV panels output

$r_{j} / x_{j} \quad$ Resistance/reactance of distribution line $j$

\section{INTRODUCTION}

$\mathrm{R}_{\mathrm{r}}^{\mathrm{E}}$ ESIDENTIAL photovoltaic (PV) panels based distributed renewable generation becomes a promising alternative power generation technology to reduce greenhouse gas emissions and promote a low-carbon lifestyle. Energy policies introduced by many countries encourage end-users to install self-consumed PV systems on their rooftops [1]. As a consequence, $\mathrm{PV}$ prosumers have emerged in large numbers [2], which are end-use consumers who can also act as energy providers if they install residential PV panels. In practice, $\mathrm{PV}$ prosumers can behave as sellers or buyers according to their net power profiles as well as electricity price when they participate in the energy sharing process. However, the proliferation of PV generators leads to the oversupply of PV energy in some areas, thus, PV curtailment may be needed when all consumers are fully supplied with local PV energy and meanwhile the wholesale market clearing prices are negative. For PV prosumers, they try to maximize the revenue from PV energy sharing to offset the investment, operation and maintenance cost of PV panels. As for the normal consumers without renewable sources, they are interested in reducing their electricity bills and some of them also concern the potential valuation of environmental benefits. Therefore, the energy sharing between PV prosumers and their nearby consumers becomes an effective approach to improve the local PV generation consumption and even reduce negative impacts caused by PV energy integration into the upstream electrical network. In this regard, from the perspective of the residential prosumers, this paper aims to optimally plan residential PV panels for maximizing the revenue of $\mathrm{PV}$ prosumers in a distribution grid via energy sharing.

The idea of energy sharing has drawn attention recently. Ref. [3] proposes a two-stage peer-to-peer energy sharing model for an energy building cluster. In Ref. [4], a novel energy sharing strategy is presented for demand-side management in a neighborhood area network (NAN), which is comprised of energy providers, users, and an electricity retailer. Ref. [5] develops a risk aversion energy sharing model based on a devised local energy market for addressing the issues caused by uncertain renewable sources from the perspective of community prosumers. In Ref. [6], a hybrid energy sharing framework of multiple distribution grids is studied with consideration of combined heat and power and demand response. Ref. [7] proposes a building welfare maximization model as well as a game-theoretical energy sharing model to investigate the effect of sharing PV electricity in an apartment building.

For optimal residential PV panels planning, Ref. [8] proposes a probabilistic dynamic allocation model to optimally determine the capacity of on-site PV generation for a residential energy hub, aiming to minimize the PV energy curtailment as well as consumer cost. In Ref. [9], a data-driven based method is proposed for the detection, verification, and estimation of residential PV system installations. Authors of Ref. [10] endeavor to find the optimal installation capacity of PV generation by splitting the budget between PV generation and energy storage for maximizing the revenue from participating in the electricity market. Ref. [11] determines the energy storage size and PV panels number for a commercial building considering heating ventilation and air conditioning systems. Ref. [12] proposes an optimal two-stage placement method for the heterogeneous distributed generators, including PV generators, in a grid-tied multi-energy microgrid with the consideration of the uncertainties from the renewable energy resources. In Ref. [13], based on a performance indicator and different parameters of the PV system, an economic study is addressed for residential PV panels installation in Santiago, Chile. Ref. [14] presents an expansion planning model of PV and battery systems for the smart house, which considers investment cost, selling price and purchasing price. However, these works only focus on energy sharing or residential PV panels planning. Up to now, the research on integrating energy sharing mechanisms with residential $\mathrm{PV}$ panels planning is still at a very early stage.

This paper develops a novel two-stage game-theoretic framework for residential PV panels planning. In the first stage, Stackelberg game theory is used to model the stochastic bi-level energy sharing problem, which is solved by a proposed descend search algorithm. In the second stage, we develop a stochastic programming based optimal power flow (OPF) model to optimally allocate residential PV panels for all PV prosumers with minimum expected active power loss. The main contributions of this paper are threefold,

1) Different from most works related to residential PV panels planning, we innovatively integrate a residential PV panels planning model with the energy sharing mechanism. To our best acknowledge, this has not been studied before. In this way, we can improve economic benefits to PV prosumers and meanwhile facilitate the installation of residential PV panels, which has practical significance.

2) Instead of directly solving the proposed bi-level energy sharing problem by using commercial solvers with Mathematical Program with Equilibrium Constraints (MPEC), we develop an efficient descend search algorithm that can significantly enhance computation efficiency. Moreover, in our proposed solution method, the feasibility of voltage constraint and current constraint is checked to ensure the reliability and security of the distribution grid operation during the energy sharing process. Thus, the proposed solution method can address both economic and operating security concerns.

3) To address the conflict of interests between PV prosumers and normal consumers, the leader-followers Stackelberg game theory is used to model a bi-level PV energy sharing problem. Besides, the uncertainties of PV energy output, load demand, as well as electricity price are simultaneously considered in this energy sharing model. 
The rest of this paper is organized as follows. In Section II, we introduce the system model, including the branch flow model, consumer agents and the coalition of PV prosumer agents. Section III describes a bi-level energy sharing problem and an efficient search algorithm-based solution method. Section IV presents the optimal PV panels allocation model with the goal of active power loss minimization. Numerical results are given in Section V. Finally, we conclude this paper in Section VI.

\section{SYSTEM MODELLING}

\section{A. Network Branch Flow Model}

In this paper, the branch flow model (BFM) [15] is used to describe the complex power flow equations of the radial electrical network. Consider a radial distribution grid $\mathcal{M}:=$ $(\mathcal{N}, \mathcal{L})$, where $\mathcal{N}:=\{0,1, \ldots, \mathcal{N}\}$ denotes the node set and $\mathcal{L}:=\{0,1, \ldots, \mathcal{L}\}$ denotes the directed line set. Except for the substation node, each node $j$ has a unique ancestor node $m$ and a set of child nodes $\mathcal{C}_{j}$. We assume the direction of the line connect node $j$ and its ancestor node $m$ is from $m$ to $j$. Note that the node $n$ is in the set of the child nodes $\mathcal{C}_{j}$ of the node $j$. Therefore, the BFM is given as follows,

$$
\begin{aligned}
& P_{m j}-r_{m j} l_{m j}+p_{j}=\sum_{n \in C_{j}} P_{j n} \quad j \in \mathrm{N} \\
& Q_{m j}-x_{m j} l_{m j}+q_{j}=\sum_{n \in C_{j}} Q_{j n} \quad j \in \mathrm{N} \\
& v_{j}-v_{m}=2\left(r_{m j} P_{m j}+x_{m j} Q_{m j}\right)-\left(r_{m j}^{2}+x_{m j}^{2}\right) l_{m j} \quad j \in \mathrm{N} \\
& l_{m j}=\frac{P_{m j}^{2}+Q_{m j}^{2}}{v_{j}} \quad j \in \mathrm{N}
\end{aligned}
$$

where the active and reactive power balances at each node are described by (1a) and (1b), respectively; $P_{m j}$ and $Q_{m j}$ represent the active and reactive power flows of line $m j$ (from the ancestor node $m$ to the node $j$ ), respectively; $p_{j}$ and $q_{j}$ denote the active and reactive power injection $(+)$ or extraction $(-)$ at the node $j$, respectively; $r_{m j}$ and $x_{m j}$ represent the resistance and reactance of line $m j$, respectively; $l_{m j}$ is the squared line current magnitude on distribution line $m j$. The voltage drop/rise on each line can be described by (1c). (1d) denotes the relationship between the power flow, squared bus voltage magnitude and squared line current magnitude, which is expressed by nonconvex. To convexify (1d), it is relaxed into an inequality as (2a) and then reformulated into a second-order cone constraint as ( $2 \mathrm{~b}$ ) by the second-order cone programming (SOCP) relaxation method [16].

$$
\begin{aligned}
& l_{m j} \geq \frac{P_{m j}^{2}+Q_{m j}^{2}}{v_{j}} \quad j \in \mathrm{N} \\
& \left\|\left(2 P_{m j}, 2 Q_{m j}, v_{j}-l_{m j}\right)\right\|_{2} \leq v_{j}+l_{m j} \quad j \in \mathrm{N}
\end{aligned}
$$

It has been proved in Ref. [17] that the relaxation is exact as long as the network is radial and the objective function of the OPF problem is strictly increasing in $l_{m j}$. In our model, the equality in (2b) also holds since we take the network loss into account and it is a strictly increasing function of $l_{m j}$.

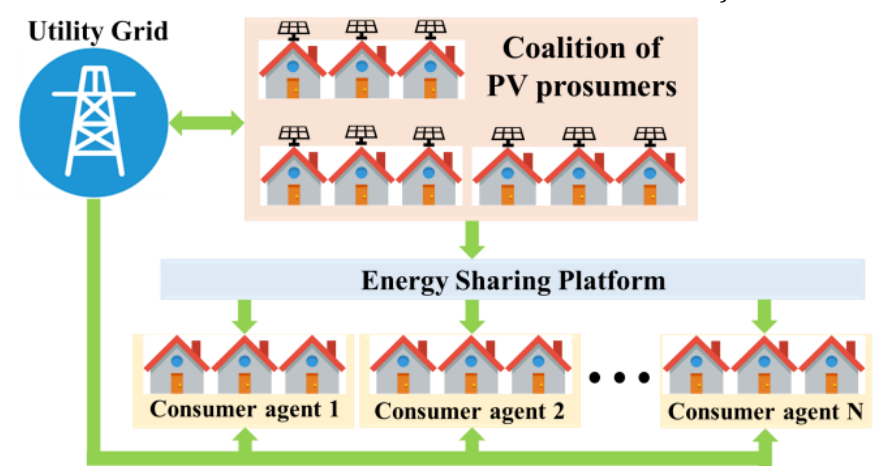

Fig. 1. The proposed energy sharing framework between the coalition and consumer agents

\section{B. Energy Sharing Agent Model}

This paper proposes a two-stage residential PV panels planning model, where the first stage is to determine the optimal sizing of PV panels by developing a Stackelberg game-based stochastic bi-level energy sharing model. The proposed energy sharing model is performed during the decision-making process of finding the total PV panel installation capacity. Besides, in the practical operation of the distribution system, our proposed bi-level energy sharing model can be used for trading energy among the coalition, consumers and the utility grid.

For better energy sharing coordination, we introduce two kinds of agents, i.e., consumer agent and prosumer agent. Specifically, the consumer agent denotes the aggregation of customers on the same node and the prosumer agent represents the aggregation of PV prosumers (owners of PV panels) on the same node. On behalf of its local consumers/prosumers, each agent is allowed to participate in the energy sharing process.

1) Revenue for the coalition of prosumer agents: In our proposed model, all prosumer agents form a coalition to maximize their common benefits by operating their residential PV panels. This assumption is based on the fact that most individual PV prosumers can only provide a small amount of $\mathrm{PV}$ energy, posing some challenges to energy management in power girds. Besides, due to the barriers to renewable energy integration [18-21], especially market entry [22], it is efficient and easy to manage the integrated PV energy. Note that the demands of the coalition are satisfied by the self-generated PV energy firstly, then the surplus PV energy is used for energy sharing with consumers. Therefore, before the coalition participates in the energy sharing process, PV energy trading among the prosumers in the coalition has been finished.

The coalition can be a seller or a buyer according to its loads and PV generations. When the coalition becomes a seller, there is a competitive relationship between the coalition and the distribution system operator (DSO) because they both try to sell electricity to the local consumers. However, when the netload of the coalition is positive, there is a dependency relationship between the coalition and the DSO since the coalition needs to purchase electricity from the utility grid via the DSO. The coalition sells its surplus PV electricity differently to the consumer agents and the utility grid to maximize its revenue. 
During the energy sharing with local consumer agents, the coalition needs to pay the bill to the energy sharing platform which acts as an intermediary agent to facilitate energy sharing. Therefore, the revenue of the coalition is defined as follows,

$$
\begin{aligned}
\operatorname{Rev}^{C}= & \sum_{i \in \mathrm{N}^{C}} \sum_{t \in \mathrm{T}}\left(1-r^{C}\right) c_{t}^{U} P_{i t}^{P V c}+\sum_{k \in \mathbb{N}^{P V}} \sum_{t \in \mathrm{T}} c_{t}^{M C P} P_{k t}^{P V g} \\
& -\sum_{k \in \mathbb{N}^{P V}} \sum_{t \in \mathrm{T}} c_{t}^{G}\left[\gamma_{t}^{L} P_{k}^{L}-\eta^{P V} \gamma_{t}^{P V} \phi^{P V} E_{s i z e}^{P V}\right]^{+} \\
& -\left(\alpha c_{i n v}^{P V}+c_{o \& m}^{P V}\right) \phi^{P V}
\end{aligned}
$$

where the first term represents the income of selling PV electricity $P_{i t}^{P V C}$ to the consumer agents with the uniform price $c_{t}^{U}$. Note that the energy sharing platform charges the coalition intermediary fee in terms of the proportion $r^{C}$ of this income. The second term is the gain of selling PV electricity $P_{i t}^{P V g}$ to the utility grid with the wholesale market clearing price $c_{t}^{M C P}$. The third term denotes that the coalition needs to buy electricity from the utility grid when its demand cannot be satisfied by its PV generation. Note that $[\cdot]^{+}$represents the projection operator onto the non-negative orthant, i.e., $[x]^{+}=\max (x, 0)$. The last term denotes the investment, operation and maintenance cost of residential PV panels. Note the daily capital recovery factor $\alpha=\frac{r^{P V}\left(1+r^{P V}\right)^{y}}{365\left(\left(1+r^{P V}\right)^{y}-1\right)}$ is to transform the investment $\cos t c_{i n v}^{P V}$ from the planning horizon into the daily horizon, where $r^{P V}$ is the interest rate and $y$ is the planning horizon.

2) Consumer agent utility function: The utility function of each consumer agent $i$ is composed of three terms as follows,

$$
U_{i}^{C}=-\sum_{t \in \mathrm{T}} c_{t}^{U} P_{i t}^{P V C}-\sum_{t \in \mathrm{T}} c_{t}^{G} P_{i t}^{G}-\sum_{t \in \mathrm{T}} w_{i}^{E} c^{E} P_{i t}^{G}
$$

where the first term and second term represent the cost of electricity purchased from the coalition and the utility grid, respectively. Generally, the conventional consumer utility function only includes the cost of purchasing electricity. However, it is not rational enough since some consumers have environmental awareness so they are interested in reducing greenhouse gas emissions. Therefore, the third term in (4) is for emissions reduction with the individual weight factor $w_{i}^{E}$. The load demand $P_{i t}^{L}$ of each consumer agent $i$ can be satisfied by buying electricity from the coalition $\left(P_{i t}^{P V c}\right)$ and/or the utility grid $\left(P_{i t}^{G}\right)$, i.e., $P_{i t}^{L}=P_{i t}^{P V c}+P_{i t}^{G}$. Thus, by replacing $P_{i t}^{G}$ in (4) by $P_{i t}^{L}-P_{i t}^{P V C}$, the willingness-to-pay (WTP) $c_{i t}^{W T P}$ for purchasing local $\mathrm{PV}$ electricity of consumer agent $i$ can be characterized as follows,

$$
c_{i t}^{W T P}=c_{t}^{G}+w_{i}^{E} c^{E} \quad i \in \mathrm{N}^{C}, t \in T
$$

Note that the individual weight factor $w_{i}^{E}$ is introduced to express the preference for emissions reduction of consumer agent $i$ : 1) $w_{i}^{E}>0$ means that the consumer agent $i$ has strong environmental awareness so his WTP $c_{i t}^{W T P}$ is higher than the real-time electricity price $c_{t}^{G}$;2) $w_{i}^{E}=0$ means that the WTP of the consumer agent $i$ equals to the real-time electricity price; 3) $w_{i}^{E}<0$ means that the environmental awareness of consumer agent $i$ is weak so his WTP is lower than the real-time electricity price.

\section{BI-LEVEL ENERGY SHARING MODEL FOR DETERMINING OPTIMAL PV PANELS INSTALLATION CAPACITY}

\section{A. Uncertainty Characterization}

In this paper, we consider three sources of uncertainties in sizing the PV panels installation capacity, i.e., PV energy output, load demand, and electricity price. The stochastic programming-based approach is employed to deal with these three uncertainty sources, which are represented by a set of representative scenarios. We assume that local historical public data of the electricity prices, loads and PV generations can be collected by the collation and a well-established backward-reduction algorithm [23] can be used to select the representatives with probabilities, which can distinguish the importance of each scenario. Each representative scenario $w \in$ $\mathcal{W}$ consists of three vectors, given as,

$$
w=\left\{\gamma_{t w}^{P V}, \gamma_{t w}^{L}, c_{t w}^{G}\right\} \quad t \in \mathrm{T}, w \in W
$$

Besides, $p_{w}$ is defined as the occurrence probability of scenario $w$, and the sum of probabilities of all considered representative scenarios equals to one, i.e., $\sum_{w \in \mathcal{W}} p_{w}=1$.

\section{B. Stackelberg Game Model}

In a leader-follower structure, the Stackelberg game is suitable for studying the decision-making processes of followers in response to the decision taken by the leaders [24]. In this paper, we model the coalition as the leader which determines the uniform price of PV electricity and participating consumer agents act as followers to purchase electricity from different sources, e.g., utility grid and coalition, according to the time-varying price. Correspondingly, the Stackelberg game is defined as follows,

$G=\left\{\left(\right.\right.$ Coalition $\left.\left.\cup N^{C}\right),\left\{\boldsymbol{c}_{t w}^{U}\right\},\left\{\boldsymbol{P}_{i t w}^{P V c}\right\},\left\{\boldsymbol{P}_{i t w}^{G}\right\},\left\{\boldsymbol{R e v}^{C}\right\},\left\{\boldsymbol{U}_{i t w}^{C}\right\}\right\}$ where (Coalition $\cup \mathcal{N}^{C}$ ) denote the player sets, the coalition acts as the leader and the consumer agents in the set $\mathcal{N}^{C}$ take the roles of followers in response to the strategy of the coalition; $\left\{\boldsymbol{C}_{t w}^{U}\right\}$ is the strategy set of the coalition; $\left\{\boldsymbol{P}_{i t w}^{P V_{c}}\right\}$ and $\left\{\boldsymbol{P}_{i t w}^{G}\right\}$ are the strategy sets of consumer agents; $\left\{\boldsymbol{R} \boldsymbol{e v} \boldsymbol{v}^{C}\right\}$ and $\left\{\boldsymbol{U}_{i t w}^{C}\right\}$ are the revenue (3) of the coalition and the utility function (4) of the consumer agent, respectively.

\section{Bi-level Energy Sharing Model}

Stackelberg game theory is adopted to model the energy sharing negotiation [25]. Here, the coalition is the leader who sets the uniform prices for PV electricity and consumer agents are the followers who react to decisions taken by the leader. Thus, the stochastic programming-based bi-level energy sharing model is formulated as follows,

$$
\begin{aligned}
\max & \operatorname{Rev}^{C}:=\sum_{w \in W} p_{w}\left[\sum_{i \in \mathrm{N}^{C}} \sum_{t \in \mathrm{T}}\left(1-r^{C}\right) c_{t w}^{U} P_{i t w}^{P V C}+\sum_{k \in \mathrm{N}^{P V}} \sum_{t \in \mathrm{T}} c_{t w}^{M C P} P_{k t w}^{P V g}\right. \\
& \left.-\sum_{k \in \mathbb{N}^{P V}} \sum_{t \in \mathrm{T}} c_{t w}^{G}\left[\gamma_{t w}^{L} P_{k}^{L}-\eta^{P V} \gamma_{t w}^{P V} \phi^{P V} E_{s i z e}^{P V}\right]^{+}\right]-\left(\alpha c_{i n v}^{P V}+c_{o \& m}^{P V}\right) \phi^{P V}
\end{aligned}
$$

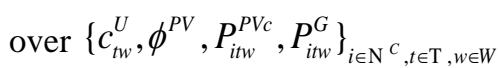

s.t. 


$$
\begin{aligned}
& \sum_{i \in \mathrm{N}^{C}} P_{i t w}^{P V c}+\sum_{k \in \mathrm{N}^{P V}} P_{k t w}^{P V g}+\sum_{k \in \mathrm{N}^{P V}} P_{k t w}^{C u r t a i l} \\
& =\left[\eta^{P V} \gamma_{t w}^{P V} \phi^{P V} E_{s i z e}^{P V}-\sum_{k \in \mathrm{N}^{P V}} \gamma_{t w}^{L} P_{k}^{L}\right]^{+} \quad t \in \mathrm{T}, w \in W \\
& \phi^{P V} E_{s i z e}^{P V} \leq E^{P V \max }, \phi^{P V} \in Z^{+} \\
& P_{i t w}^{P V c}, P_{k t w}^{P V g}, P_{k t w}^{C u r t a i l} \geq 0 \\
& i \in \mathrm{N}^{C}, k \in \mathrm{N}^{P V}, t \in \mathrm{T}, w \in W \\
& \max U_{i t w}^{C}:=-c_{t w}^{U} P_{i t w}^{P V c}-c_{t w}^{W T P} P_{i t w}^{G} \\
& \text { over }\left\{P_{i t w}^{P V c}, P_{i t w}^{G}\right\}_{i \in \mathrm{N}}{ }^{C}, t \in \mathrm{T}, w \in W \\
& \text { s.t. } P_{i t w}^{P V c}+P_{i t w}^{G}=\gamma_{t w}^{L} P_{i}^{L}: \lambda_{i t w}^{L} \quad i \in \mathrm{N}^{C}, t \in T, w \in W \\
& \quad P_{i t w}^{P V C} \geq 0: \mu_{i t w}^{P V c} \quad i \in \mathrm{N}^{C}, t \in T, w \in W \\
& \quad P_{i t w}^{G} \geq 0: \mu_{i t w}^{G} \quad i \in \mathrm{N}^{C}, t \in T, w \in W
\end{aligned}
$$

where the upper-level problem (6a)-(6h) is to maximize revenue over considered time periods in all scenarios. (6b) describes the dispatch of surplus PV electricity, which can be either fed into consumer agents $\left(P_{i t w}^{P V_{c}}\right)$, sold to the utility grid $\left(P_{k t w}^{P V g}\right)$, or curtailed $\left(P_{k t w}^{\text {Curtail }}\right)$. Note that the right side of $(6 \mathrm{~b})$ can be defined as the surplus PV electricity that can be supplied to the energy sharing process. It should be noted that PV energy curtailment becomes an optimal option in some time periods where all consumer agents are fully provided with local PV generation and the wholesale market clearing prices are negative [26]. For security reasons, (6c) is added to ensure that total PV panels installation capacity cannot exceed the PV hosting capacity of the grid. (6d) guarantees that the upper-level variables are non-negative.

The lower-level problem (6e)-(6h) aims to maximize the utility function of each consumer agent in each time period and scenario. (6f) balances the supply and demand for each consumer agent node. In (6g) and (6h), the decision variables of this lower-level problem are limited to positive values. Since the lower-level problem is linear and continuous, it can be substituted by Karush-Kuhn-Tucker (KKT) conditions [27]. To facilitate the understanding of readers, the proposed bi-level energy sharing model (6) can be written as follows,

$$
\begin{aligned}
\min _{\{x, y, \lambda, \mu\}} & f_{1}(x, y, \lambda, \mu) \\
\text { s.t. } & h_{1}(x, y, \lambda, \mu)=0 \\
& g_{2}(x, y, \lambda, \mu) \geq 0 \\
\min _{\{y, \lambda, \mu\}} & f_{2}(x, y) \\
\text { s.t. } & h_{2}(x, y)=0: \lambda \\
& g_{2}(x, y) \geq 0: \mu
\end{aligned}
$$

The KKT conditions of the lower-level problem (7d)-(7f) can be implemented in the upper-level problem (7a)-(7c), given as follows,

$$
\begin{aligned}
\min _{\{x, y, \lambda, \mu\}} & f_{1}(x, y, \lambda, \mu) \\
\text { s.t. } & h_{1}(x, y, \lambda, \mu)=0 \\
& g_{2}(x, y, \lambda, \mu) \geq 0 \\
& \nabla_{y} f_{2}(x, y)+\lambda \nabla_{y} h_{2}(x, y)+\mu \nabla_{y} g_{2}(x, y)=0 \\
& h_{2}(x, y)=0
\end{aligned}
$$

$$
g_{2}(x, y) \geq 0 \perp \mu \geq 0
$$

The Lagrangian is introduced as follows,

$$
\begin{aligned}
L= & -c_{t w}^{U} P_{i t w}^{P V C}-c_{t w}^{W T P} P_{i t w}^{G}-\lambda_{i t w}^{L}\left(P_{i t w}^{P V C}+P_{i t w}^{G}-P_{i t w}^{L}\right) \\
& -\mu_{i t w}^{P V c} P_{i t w}^{P V c}-\mu_{i t w}^{G} P_{i t w}^{G}
\end{aligned}
$$

Therefore, the lower-level problem can be replaced by KKT conditions, described as follows,

$$
\begin{aligned}
& \frac{\partial L}{\partial \lambda_{i t w}^{L}}=P_{i t w}^{P V C}+P_{i t w}^{G}-P_{i t w}^{L}=0 \\
& \frac{\partial L}{\partial P_{i t w}^{P V C}}=c_{i t w}^{W T P}-\lambda_{i t w}-\mu_{i t w}^{P V c}=0 \\
& \frac{\partial L}{\partial P_{i t w}^{G}}=c_{i t w}^{W T P}-\lambda_{i t w}-\mu_{i t w}^{G}=0 \\
& P_{i t w}^{P V c} \geq 0 \perp \mu_{i t w}^{P V c} \geq 0 \\
& P_{i t w}^{G} \geq 0 \perp \mu_{i t w}^{G} \geq 0
\end{aligned}
$$

Then the stochastic bi-level energy sharing model (6) can be formulated as mathematical programming with equilibrium constraints (MPEC) model [28], as follows,

$$
\begin{array}{r}
\max \operatorname{Rev}^{C}:=\sum_{w \in W} p_{w}\left[\sum_{i \in \mathrm{N}^{c}} \sum_{t \in \mathrm{T}}\left(1-r^{C}\right) c_{t w}^{U} P_{i t w}^{P V C}+\sum_{k \in \mathrm{N}^{P V}} \sum_{t \in \mathrm{T}} c_{t w}^{M C P} P_{k t w}^{P V g}\right. \\
\left.-\sum_{k \in \mathrm{N}^{P V}} \sum_{t \in \mathrm{T}} c_{t w}^{G}\left[\gamma_{t w}^{L} P_{k}^{L}-\eta^{P V} \gamma_{t w}^{P V} \phi^{P V} E_{s i z}^{P V}\right]^{+}\right]-\left(\alpha c_{i n v}^{P V}+c_{o \& m}^{P V}\right) \phi^{P V}
\end{array}
$$

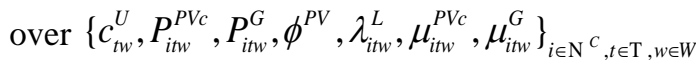

s.t. $(6 \mathrm{~b})-(6 \mathrm{~d}),(10 \mathrm{a})-(10 \mathrm{e})$

\section{D.Linearization of Bi-level Energy Sharing Model}

The MPEC model (11) contains two nonlinearities, 1) the nonlinear term $c_{t w}^{U} P_{i t w}^{P V c}$ in the objective function $\left.(11 \mathrm{a}) ; 2\right)$ the complementarity constraints (10d) and (10e). This may make the problem unsolvable. Therefore, the original model (11) needs to be linearized. Firstly, the strong duality condition can be used to obtain a linear expression for $c_{t w}^{U} P_{i t w}^{P V C}$. As stated in the strong duality theorem [29], if a problem is convex, the objective functions of the primal and dual problems have the same value at the optimum. Therefore, the primary objective function (6e) of the lower-level problem is equal to its dual objective function, as follows,

$$
c_{t w}^{U} P_{i t w}^{P V c}+c_{t w}^{W T P} P_{i t w}^{G}=\lambda_{i t w}^{L} \gamma_{t w}^{L} P_{i}^{L}
$$

Then we can obtain the following expression for the nonlinear term $c_{t w}^{U} P_{i t w}^{P V c}$,

$$
c_{t w}^{U} P_{i t w}^{P V c}=\lambda_{i t w}^{L} \gamma_{t w}^{L} P_{i}^{L}-c_{t w}^{W T P} P_{i t w}^{G}
$$

Secondly, the complementarity constraints (10d) and (10e) can be linearized using linear expressions proposed in Ref. [30], so the following constraints should be added,

$$
\begin{aligned}
& P_{i t w}^{P V C}, P_{i t w}^{G} \geq 0 \quad i \in \mathrm{N}^{C}, t \in \mathrm{T}, w \in W \\
& \mu_{i t w c}^{P V C}, \mu_{i t w}^{G} \geq 0 \quad i \in \mathrm{N}^{C}, t \in \mathrm{T}, w \in W \\
& P_{i t w}^{P V C} \leq\left(1-u_{i t w}^{P V c}\right) M \quad i \in \mathrm{N}^{C}, t \in \mathrm{T}, w \in W \\
& P_{i t w}^{G} \leq\left(1-u_{i t w}^{G}\right) M \quad i \in \mathrm{N}^{C}, t \in \mathrm{T}, w \in W \\
& \mu_{i t w}^{P V c} \leq u_{i t w}^{P V c} M \quad i \in \mathrm{N}^{C}, t \in \mathrm{T}, w \in W
\end{aligned}
$$




$$
\begin{aligned}
& \mu_{i t w}^{G} \leq u_{i t w}^{G} M \quad i \in \mathrm{N}^{C}, t \in \mathrm{T}, w \in W \\
& u_{i t w}^{P V c}, u_{i t w}^{G} \in\{0,1\}
\end{aligned}
$$

Finally, the linearized MPEC model can be written as follows,

$$
\begin{aligned}
& \max \sum_{w \in W} p_{w}\left[\sum_{i \in \mathrm{N}^{C}} \sum_{t \in \mathrm{T}}\left(1-r^{C}\right)\left(\lambda_{i t w}^{L} \gamma_{t w}^{L} P_{i}^{L}-c_{i t w}^{W T P} P_{i t w}^{G}\right)\right. \\
& \left.+\sum_{k \in \mathrm{N}^{P V}} \sum_{t \in \mathrm{T}} c_{t w}^{M C P} P_{k t w}^{P V g}-\sum_{k \in \mathrm{N}^{P V}} \sum_{t \in \mathrm{T}} c_{t w}^{G}\left[\gamma_{t w}^{L} P_{k}^{L}-\eta^{P V} \gamma_{t w}^{P V} \phi^{P V} E_{s i z e}^{P V}\right]^{+}\right] \\
& -\left(\alpha c_{i n v}^{P V}+c_{o \& m}^{P V}\right) \phi^{P V}
\end{aligned}
$$

$$
\text { over }\left\{c_{t w}^{U}, P_{i t w}^{P V c}, P_{i t w}^{G}, \phi^{P V}, \lambda_{i t w}, \mu_{i t w}^{P V c}, \mu_{i t w}^{G}\right\}_{i \in \mathrm{N}{ }^{c}, t \in \mathrm{T}, w \in W}
$$$$
\text { s.t. (6b)-(6h), (10a)-(10c), (14a)-(14g) }
$$

\section{E. Descend Search based Solution Algorithm}

Although the linearized MPEC model (15) can be directly solved by cutting-edge solvers, such as CLPEX [31] and Gurobi [32], its computation burden may be quite heavy due to a very large number of scenarios and mixed-integer variables. In this regard, this paper develops an efficient solution algorithm for solving the linearized MPEC model.

As the leader in the Stackelberg game, the coalition sets the uniform price for its PV electricity sold to the consumer agent. According to the definition stated in Ref. [25], the bi-level energy sharing model (6) reaches the Stackelberg Equilibrium (SE) when all players acquire the optimal solutions. Therefore, our proposed game-theoretic framework reaches a SE as long as the coalition finds the optimal uniform price and meanwhile all consumer agents choose their electricity consumption. In this paper, it is assumed the coalition knows the load curves of all the consumer agents in the paper. This assumption is reasonable in practice since the coalition can acquire the load information of each consumer agent by the long-term observation via the nonintrusive load monitoring [33]. Besides, the consumer agent is on behalf of all consumers connected to the same node, so the exact load demand of each consumer cannot be obtained by the coalition. In this regard, the privacy of individual consumers can be protected. Therefore, the optimal uniform price set by the coalition is equal to the WTP offered by the consumer agents. Thus, the coalition prefers to sell its surplus PV electricity to the consumer agent with the highest WTP, followed by the consumer agent with the second-highest WTP, etc. Thus, the surplus PV electricity will be dispatched in the descending order by the WTP of consumer agents. Besides, because the wholesale market clearing price is always lower than the WTP, so the coalition prefers to sell its PV electricity firstly to the consumer agents and the second to the utility grid. Note that for the extreme situation where the uniform price equals the electricity price, we assume that the local consumers prefer to purchase the electricity from the collation firstly due to their environmental awareness. This can facilitate the utilization of local PV energy and reduce the negative impacts on the utility grid caused by PV energy integration.

In this regard, we develop a descend search algorithm to calculate the uniform price, as shown in Algorithm 2. The idea
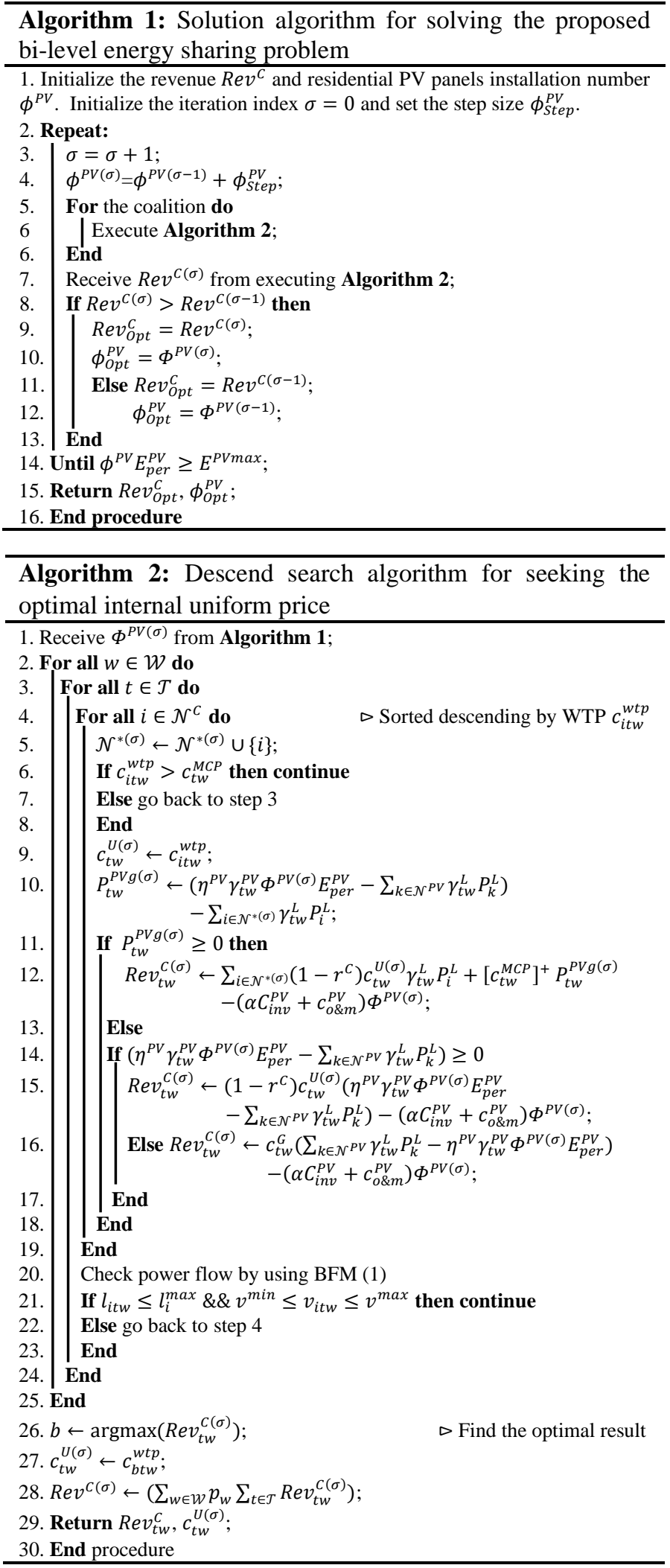

of this search algorithm is to iteratively update the revenue from selling surplus PV electricity to consumer agents, whereby the consumer agents are ranked in descending order by their WTP. Finally, the uniform price that brings about the highest revenue to the coalition will be returned. Therefore, the optimal uniform price can be found by using Algorithm 2 


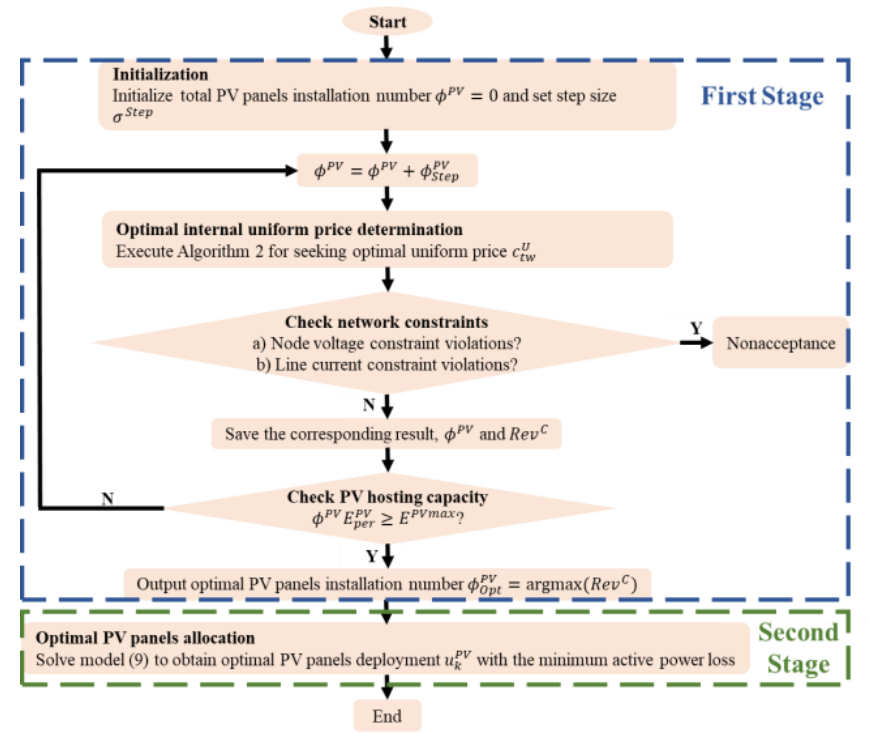

Fig. 2. Flowchart of our proposed two-stage game-theoretic residential PV panels planning framework

instead of directly solving the problem (8). Moreover, to ensure the reliability and security of the distribution grid during the energy sharing process, the feasibility voltage constraint and current constraint are checked by using the BFM. Our proposed descend search algorithm-based solution method is given as Algorithm 1. Besides, Fig. 2. is plotted to depict the flowchart of our proposed two-stage game-theoretic residential PV panels planning framework.

\section{IV.Stochastic Optimal PV Panels Allocation IN the COALITION OF PROSUMER AGENTS}

After the first stage, the optimal installation capacity of residential PV panels for the coalition can be obtained. Then the second stage of our proposed two-stage planning framework concerns the optimal residential PV panels allocation among the prosumer agents of the collation. At this stage, the power loss becomes the most important variable since it changes in terms of the allocation result of PV panels. The primary goal of the second-stage model is to minimize active power loss so that economic loss can be reduced. The stochastic optimal residential PV panels allocation model is as follows,

$$
\min \sum_{w \in W} p_{w} \sum_{t \in \mathrm{T}} \sum_{j \in \mathrm{N}} r_{m j} l_{m j w w}
$$

over

$$
\begin{aligned}
& \left\{P_{m k t w}, Q_{m k t w}, P_{m i t w}, Q_{m i t w}, v_{j t w}, l_{m j t w}, u_{k}^{P V}\right\}_{k \in \mathrm{N}^{P V}, i \in \mathrm{N}^{C}, t \in \mathrm{T}, w \in W, j \in \mathrm{N}} \\
& \text { s.t. } \\
& P_{m k t w}-r_{m k} l_{m k t w}+\eta^{P V} \gamma_{t w}^{P V} u_{k}^{P V} E_{s i z e}^{P V}-\gamma_{t w}^{L} P_{k}^{L}=\sum_{n \in C_{k}} P_{k n t w} \\
& k \in \mathrm{N}^{P V}, t \in \mathrm{T}, w \in W \\
& P_{m i t w}-r_{m i} l_{m i t w}-\gamma_{t w}^{P V} P_{i}^{L}=\sum_{n \in C_{i}} P_{i n t w} \quad i \in \mathrm{N}^{C}, t \in \mathrm{T}, w \in W \\
& Q_{m k t w}-x_{m k} l_{m k t w}-\gamma_{t w}^{L} Q_{k}^{L}=\sum_{n \in C_{k}} Q_{k n t w} \quad k \in \mathrm{N}^{P V}, t \in \mathrm{T}, w \in W
\end{aligned}
$$

$$
\begin{aligned}
& Q_{m i t w}-x_{m i} l_{m i t w}-\gamma_{t w}^{L} Q_{i}^{L}=\sum_{n \in C_{i}} Q_{i n t w} \quad i \in \mathrm{N}^{C}, t \in \mathrm{T}, w \in W \\
& v_{j t w}-v_{m t w}=2\left(r_{m j} P_{m j t w}+x_{m j} Q_{m j w}\right)-\left(r_{m j}^{2}+x_{m j}^{2}\right) l_{m j w w} \\
& j \in \mathrm{N}, t \in \mathrm{T}, w \in W \\
& \left\|\left(2 P_{m j w}, 2 Q_{m j w}, v_{j t w}-l_{m j j w}\right)\right\|_{2} \leq v_{j t w}+l_{m j w} \\
& j \in \mathrm{N}, t \in \mathrm{T}, w \in W \\
& v^{m i n} \leq v_{j t w} \leq v^{\max } \quad j \in N, t \in \mathrm{T}, w \in W \\
& 0 \leq l_{m j w w} \leq l_{m j}^{\max } \quad j \in \mathrm{N}, t \in \mathrm{T}, w \in W \\
& u_{k}^{P V} \leq u_{k}^{P V \max } \quad k \in \mathrm{N}^{P V} \\
& \sum_{k \in \mathrm{N}^{P V}} u_{k}^{P V}=\phi^{P V}, u_{k}^{P V} \in Z^{+}
\end{aligned}
$$

where the objective function (16a) is to minimize the expected active power loss of all considered uncertainty scenarios. (16b) and (16c) describe the active power balance on the node with the prosumer agent and consumer agent, respectively. (16d) and (16e) describe the reactive power balance on each node. (16f) and (16f) are derived from (1c) and (2), respectively. (16h) is the voltage constraint and (16i) is the current constraint. (16j) guarantees that the residential PV panels installation number at each prosumer agent node cannot exceed its maximum allowable installation number, which is determined by the installation conditions. (16k) ensures that the total installation number of residential PV panels equals the optimal value obtained from the first stage.

\section{V.NUMERICAL RESULTS}

\section{A. Implementation on IEEE 33-node Distribution System}

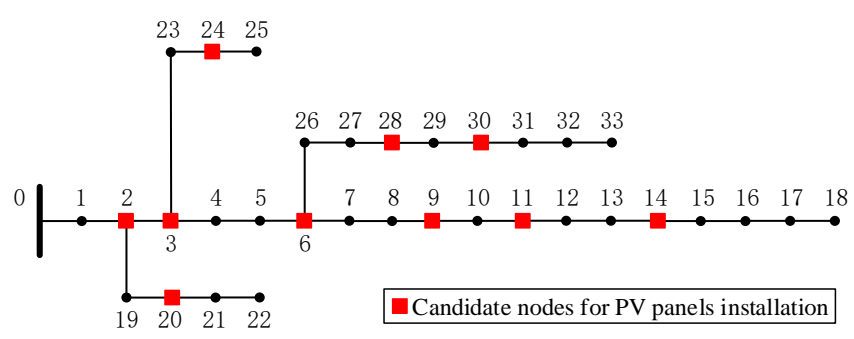

Fig. 3. IEEE 33-node distribution grid with candidate nodes for PV panels installation

In this section, we employ the modified IEEE 33-node distribution grid to test our proposed residential PV panels planning model. Detailed information on this test system can be referred to [34]. Fig. 4 depicts $125(5 * 5 * 5)$ uncertainty scenarios, consisting of five load scenarios, five PV output scenarios and five electricity price scenarios. For the sake of simplicity, the electricity price $c_{t w}^{G}$ is assumed to be equal for all agents in the test system and it consists of the market clearing price $c_{t w}^{M C P}$, generation markup $(0.013 \$ / \mathrm{kWh})$ and delivery charge $(0.036 \$ / \mathrm{kWh})$ [35], i.e., $c_{t w}^{G}=c_{t w}^{M C P}+$ $0.013+0.036 \$ / \mathrm{kWh}$. (Source: Power Smart Pricing). Due to the characteristic of residential PV panels output, the length of the entire time period $\mathcal{T}$ is thirteen hours (6:00-18:00) and the duration of each time period is one hour. We randomly assume 
the following weight factors to describe different consumer agent preferences on WTP: $w_{i}^{E}=[6.3,8.2,-7.5,8.3,2.6,-8$, $-4.4,0.9,9.2,9.3,-6.8,9.4,9.1,-0.3,6,-7.2,-1.6,8.3,5.8,9.2$, $3.1,-9.3,7]$ for consumer agents $1,4,5,7,8,10,12,13,15,16$, $17,18,19,21,22,23,25,26,27,29,31,32$ and 33, respectively. Note that these weight factors have significant effects on optimal residential PV panels planning results and in practice, they can be adjustable depending on the preferences of consumer agents on a case by case basis. Other parameters including greenhouse gas emission price, per residential PV panel size, and residential PV panel investment, maintenance and operation cost are summarized in Table I. Table II lists the optimal residential PV panels allocation result of our proposed two-stage game-theoretic model. All case studies are implemented by using MATLAB on a computer with an Intel Core $i 7$ of $2.4 \mathrm{GHz}$ and $12 \mathrm{~GB}$ memory.

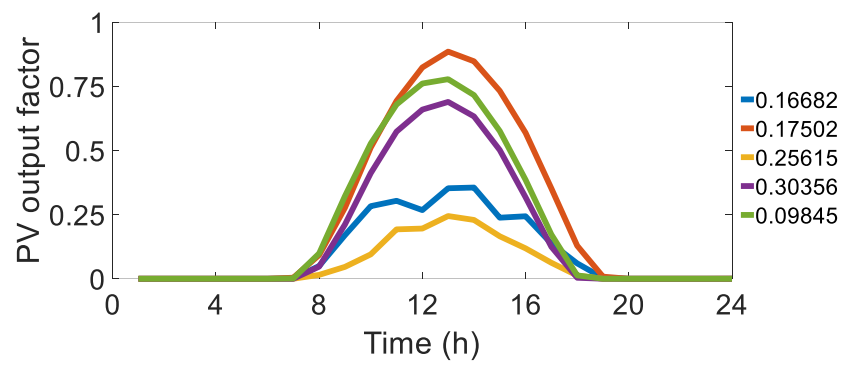

a)

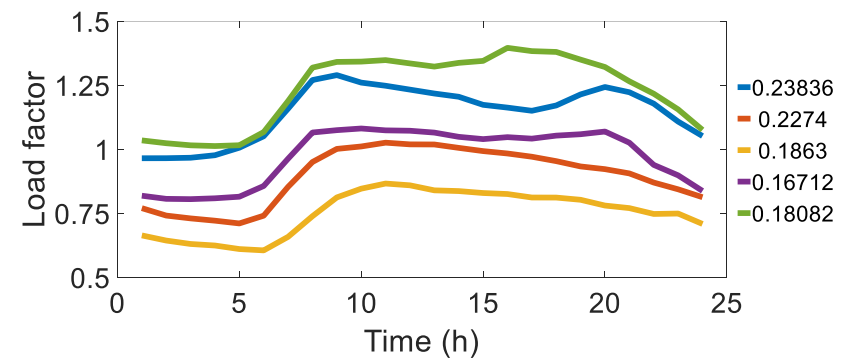

b)

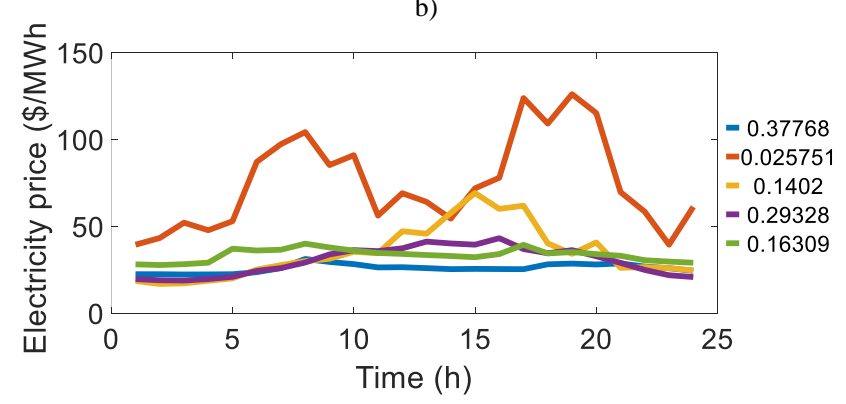

c)

Fig. 4. Uncertainty scenarios of a) load factor $\gamma_{t w}^{L}$, b) PV output factor $\gamma_{t w}^{P V}$, and c) electricity price $c_{t w}^{G}$

TABLE I

ReLATED PARAMETERS IN THE 33-NODE CASE

\begin{tabular}{ll}
\hline Parameter name & Parameter value \\
\hline Greenhouse gas emission price & $0.0125 \$ / \mathrm{kWh}[36]$ \\
Per residential PV panel size & $200 \mathrm{kVA}[37]$ \\
Residential PV panel planning cost & $3.05 \$ / \mathrm{W}[37]$ \\
Residential PV panel output efficiency & $95 \%[37]$ \\
Planning horizon & 20 years \\
Depreciation rate of PV panels & $5 \%$ \\
\hline
\end{tabular}

TABLE II

OPtimal RESIDENTIAL PV PANELS PlANNING RESUlt IN 33-NODE SYSTEM

\begin{tabular}{cc}
\hline Candidate locations (nodes) & PV panels capacity (kVA) \\
\hline 2 & 148.4 \\
3 & 129 \\
6 & 259 \\
9 & 269 \\
11 & 63.2 \\
14 & 165.6 \\
20 & 151.8 \\
24 & 218.6 \\
28 & 240 \\
30 & 255.4 \\
\hline
\end{tabular}

\section{1) From the Perspective of the Coalition}

TABLE III

RESIDENTIAL PV PANELS PLANNING SIZE AND COST AND DAILY REVENUE WITH AND WITHOUT ENERGY SHARING IN 33-NODE TEST SYSTEM

\begin{tabular}{lll}
\hline Energy sharing mechanism & No & Yes \\
Residential PV panels planning size & $6.4 * 10_{2} \mathrm{kVA}$ & $1.9 * 10_{3} \mathrm{kVA}$ \\
Residential PV panels planning cost & $1.45^{*} 0_{6} \$$ & $4.3 * 10_{6} \$$ \\
Daily revenue & $32.45 \$$ & $110.27 \$$ \\
\hline
\end{tabular}

In this subsection, we demonstrate the results from the perspective of the coalition. Table III lists comparisons on the PV panels planning size and cost as well as the daily revenue with and without energy sharing. We can see from this table that the PV panels installation capacity with energy sharing is more than that without consideration of energy sharing, so its corresponding PV panels planning cost is higher. This is because local consumer agents with good environmental awareness would like to purchase PV electricity from the coalition, so more PV panels will be invested. Besides, it also can be observed that daily revenue improves significantly after energy sharing between the coalition and consumer agents, which can bring approximately 77.8 \$ revenue improvement each day.

Fig. 5 illustrates the hourly revenue of the coalition in representative scenario 6 and scenario 12. As shown in this figure, hourly revenue is negative in the morning and becomes positive from noon. The main reason is that the residential PV panels only can provide a small amount of PV electricity in the morning, but the PV production is sufficient at midday. Fig. 6 depicts the dispatch of PV electricity provided by the coalition in representative scenario 4 . It can be seen that the coalition sells more PV electricity to the consumer agents in each time period. The coalition allocates the PV electricity in a way to maximize its revenue, so it sells PV electricity differently to the utility grid and the consumer agents in each time period. According to the WTP equation (5), we can know that the wholesale market clearing price $c_{t w}^{M C P}$ is always lower than the WTP $c_{i t w}^{W T P}$, so the coalition prefers to sell its PV electricity firstly to the consumer agents and then to the utility grid. Fig. 7 shows the hourly optimal uniform price in representative scenario 15. It can be observed from this figure that the uniform price is high in time periods of non-peak PV generation (6:00-10:00 and 16:00-18:00) and relatively low in time periods of peak PV generation (10:00-16:00). This is because the PV electricity can be consumed by local consumers during 
the daytime, the coalition sets low uniform prices so its PV electricity can be dispatched to more consumer agents. While in the morning or early night, the coalition would like to choose a high uniform price to maximize its revenue with low PV production.

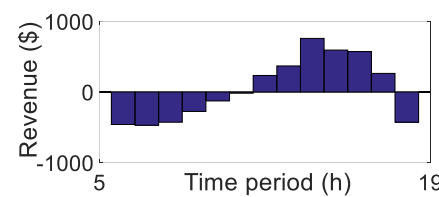

a) scenario 6

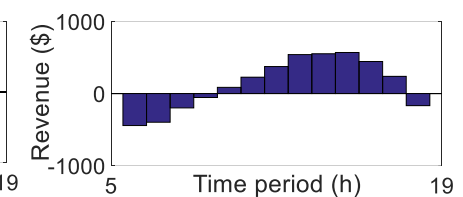

b) scenario 12
Fig. 5. Hourly revenue of the coalition in representative a) scenario 6 and b) scenario 12

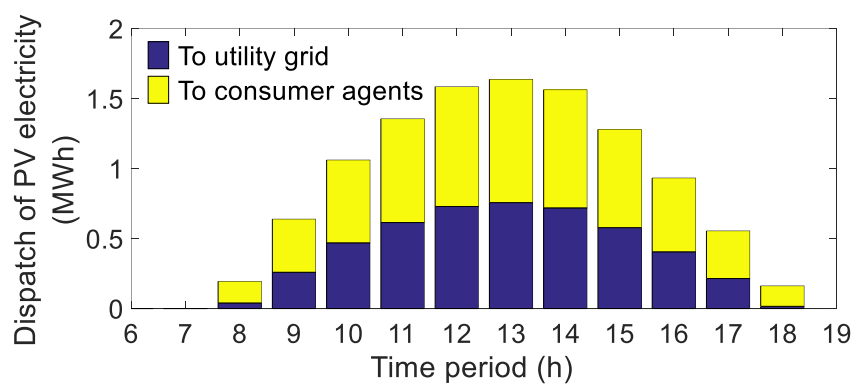

Fig. 6. PV generation sold to the consumer agents and sold to the utility grid in representative scenario 4

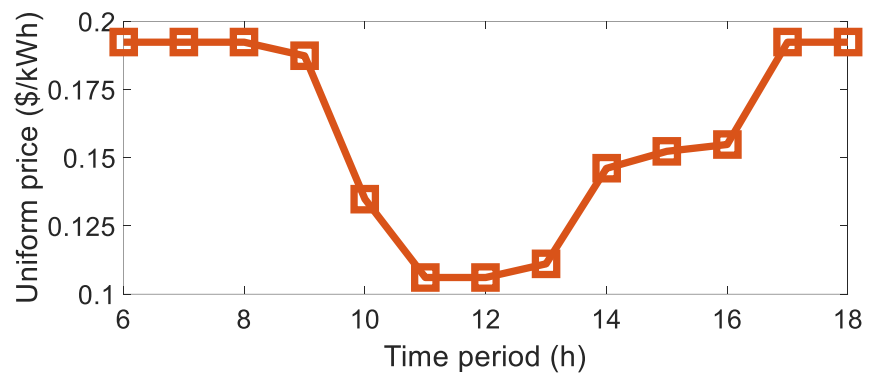

Fig. 7. Optimal uniform price of each time period in representative scenario 15

TABLE IV

Computation Performance on SOlving Proposed Bi-LeVel EnERgy SHARING PROBLEM BY DIFFERENT METHODS CONSIDERING DIFFERENT NUMBER OF OPERATION SCENARIOS

\begin{tabular}{ccc}
\hline \multirow{2}{*}{ No. of operation scenarios } & \multicolumn{2}{c}{ Computation time (sec) } \\
\cline { 2 - 3 } & MILP method & Proposed method \\
\hline $1(1 * 1 * 1)$ & 2.91 & 4.38 \\
$8(2 * 2 * 2)$ & 120.57 & 20.05 \\
$27(3 * 3 * 3)$ & 3961.12 & 67.66 \\
$64(4 * 4 * 4)$ & - & 160.38 \\
$125(5 * 5 * 5)$ & - & 313.25 \\
\hline
\end{tabular}

To investigate the computation performance on our proposed solution method (see Algorithm 1 and Algorithm 2) for solving energy sharing problem (6), the conventional mixed-integer linear programming (MILP) method for solving our formulated MPEC problem (11) is used as the benchmark. Table IV lists the computation time of solving the proposed bi-level energy sharing problem by these two methods considering the different number of operation scenarios. As shown in this table, both two solution methods can be used to solve the proposed problem with low considered scenario numbers (e.g. 1, 8, 27) while our proposed method shows a clear advantage over the first method. With high scenarios numbers (e.g. 64, 125), a huge computation burden may be involved so the commercial solver cannot handle the original problem. By contrast, our solution algorithm can solve the same problem with acceptable computation time. Note that the solution obtained by these two methods satisfies all conditions in each considered scenario, so the solution becomes more robust with the consideration of more operation scenarios [38]. In this regard, the robustness and reliability of the solution obtained by our proposed solution method can be more guaranteed since this method can take a large number of uncertainty scenarios into account.

2) The Tradeoff between Total Residential PV Panels Size and the Daily Coalition Revenue

In this subsection, a sensitivity analysis of the total residential PV panels size with the daily coalition revenue is performed. Fig. 8 depicts the tradeoff curve between the total residential PV panel size and the daily coalition revenue with and without energy sharing, respectively. With energy sharing, fast-growing daily revenue can be observed with increasing residential $\mathrm{PV}$ panels size until the investment capacity reaches about $1,900 \mathrm{kVA}$, then the daily revenue declines after more residential PV panels installation. While without energy sharing, the daily coalition revenue shows a rapid increase at first as increasing residential PV panels installation capacity, then it becomes fluctuant and finally performs a gradual decrease. This is because larger residential PV panels investment leads to high planning costs, which cannot be covered by the economic benefit of PV energy sharing.

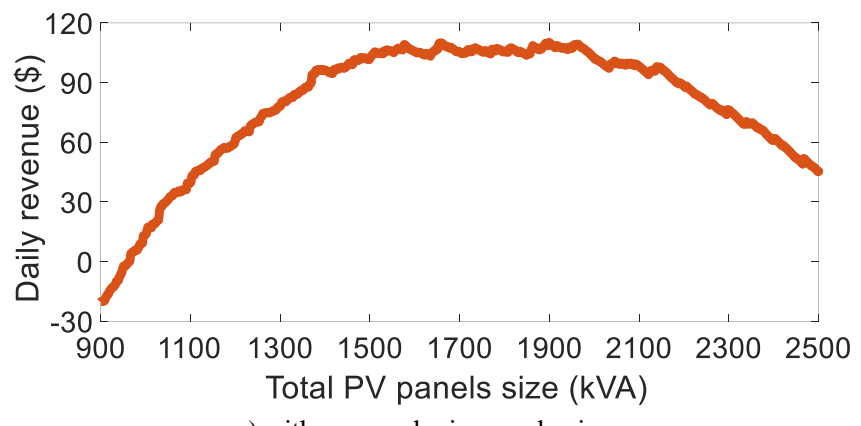

a) with energy sharing mechanism

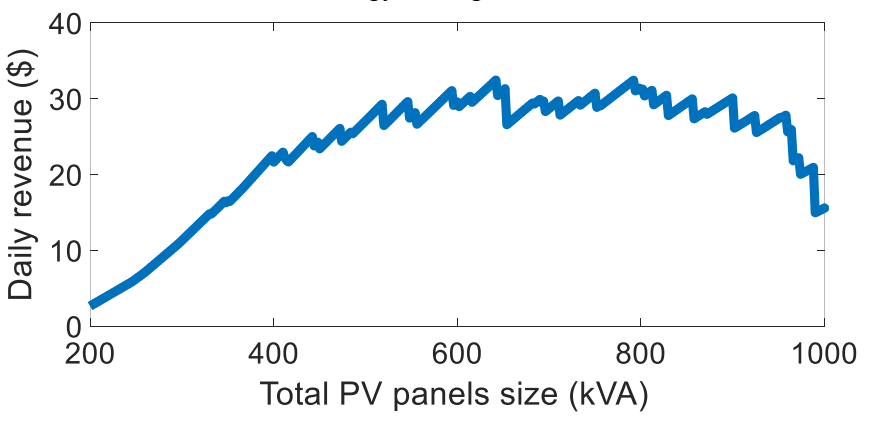

b) without energy sharing mechanism

Fig. 8. Tradeoff curve between the total PV panel size and the daily coalition revenue a) with and b) without energy sharing mechanism in the 33-node system.

\section{B. Implementation on IEEE 123-node Distribution System}

The IEEE 123-node distribution grid [39] is adopted as the large-scale test system to verify the proposed planning model 
and solution algorithm. In this case, uncertainty scenarios including load factor, PV energy output factor, and electricity price are the same as those in the 33-node case. Other parameters including greenhouse gas emission price, per residential PV panel size, and residential PV panel investment, maintenance and operation cost are also the same as those in the 33-node case.

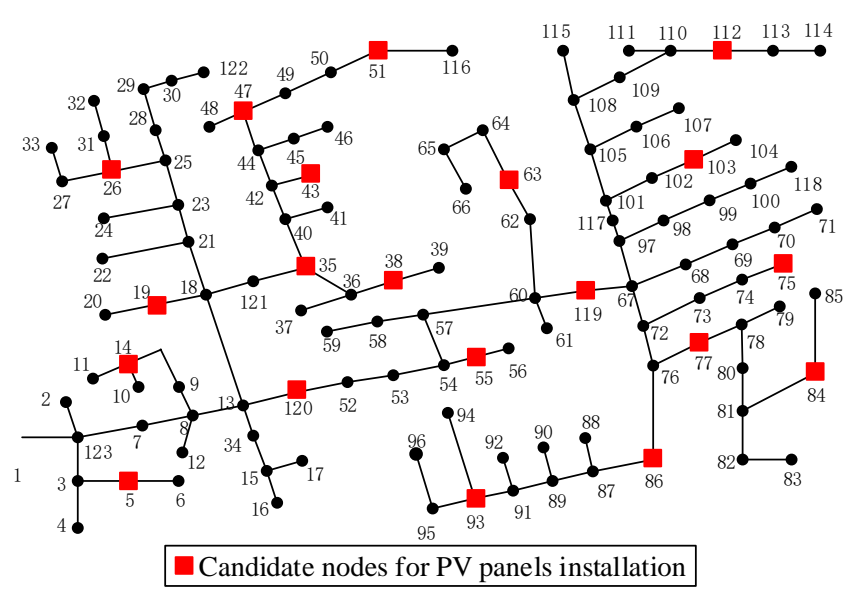

Fig. 9. IEEE 123-node distribution grid with candidate nodes for PV panels installation

TABLE V

Optimal Residential PV PANELS PLANNING RESUlt In 123-NODE SYSTEM

\begin{tabular}{cc|cc}
\hline $\begin{array}{c}\text { Locations } \\
\text { (nodes) }\end{array}$ & $\begin{array}{c}\text { PV panels capacity } \\
\text { (kVA) }\end{array}$ & $\begin{array}{c}\text { Locations } \\
\text { (nodes) }\end{array}$ & $\begin{array}{c}\text { PV panels capacity } \\
\text { (kVA) }\end{array}$ \\
\hline 5 & 298.67 & 63 & 279.10 \\
14 & 97.37 & 75 & 64.01 \\
19 & 663.21 & 77 & 386.75 \\
26 & 394.31 & 84 & 241.06 \\
35 & 87.65 & 86 & 634.42 \\
38 & 222.52 & 93 & 444.33 \\
43 & 67.30 & 103 & 305.76 \\
47 & 96.56 & 112 & 70.35 \\
51 & 139.54 & 119 & 15.68 \\
55 & 1300.40 & 120 & 191.07 \\
\hline
\end{tabular}

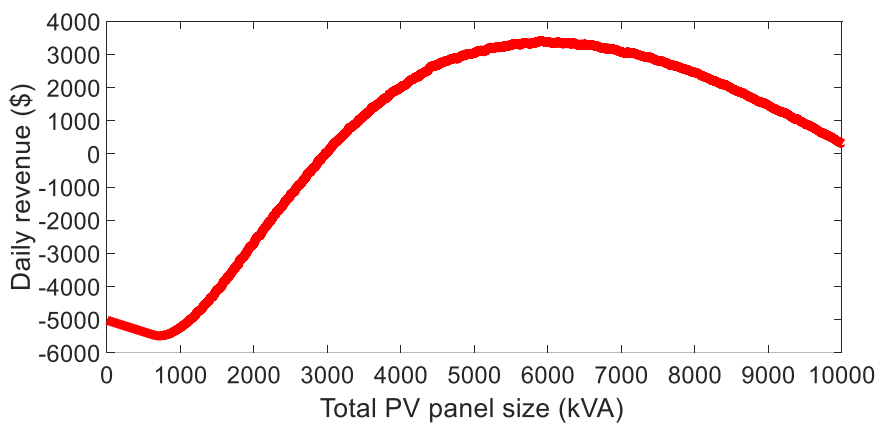

Fig. 10. Tradeoff curve between the total PV panel size and the daily coalition revenue with energy sharing in the 123-node system.

Fig. 9 depicts this modified test system by selecting twenty candidate nodes for PV panels installation, i.e., 5, 14, 19, 26, $35,38,43,47,51,55,63,75,77,84,86,93,103,112,119$ and 120 . Besides, random weight factors in the range of $[-10,10]$ are assumed to define the consumer agent preferences on WTP. Residential PV panels planning result is listed in Table V. Fig. 10 is plotted to demonstrate the tradeoff between the total residential PV panel size and the daily coalition revenue with the energy sharing mechanism. As shown in this figure, the daily revenue rises rapidly with the increase of the PV panel size. After the PV panel size reaches approximately 6,000 kVA, the daily revenue decreases with the increasing PV panel size.

\section{VI.CONCLUSION}

In this paper, we develop a novel two-stage game-theoretic framework of residential PV panels planning. In the first stage, Stackelberg game theory is used to model the stochastic bi-level energy sharing problem, which is solved by our proposed descend search algorithm-based solution method, so the optimal installation capacity of residential PV panels can be obtained. In the second stage, we develop a stochastic programming-based optimal OPF model to optimally allocate residential PV panels for all PV prosumers with minimum expected active power loss. Finally, the IEEE 33-node and 123-node test systems are used to demonstrate the effectiveness of our proposed framework. As shown in numerical results, with the consideration of energy sharing mechanism during the PV panels planning stage, not only economic benefits to PV prosumers can be improved but also the residential PV panels installation is facilitated.

\section{REFERENCES}

[1] E. McKenna and M. Thomson, "Photovoltaic metering configurations, feed-in tariffs and the variable effective electricity prices that result," IET Renewable Power Generation, vol. 7, no. 3, pp. 235-245, 2013.

[2] J. Li, C. Zhang, Z. Xu, J. Wang, J. Zhao, and Y.-J. A. J. Zhang, "Distributed transactive energy trading framework in distribution networks," IEEE Transactions on Power Systems, vol. 33, no. 6, pp. 7215-7227, 2018.

[3] S. Cui, Y.-W. Wang, and J.-W. J. Xiao, "Peer-to-Peer Energy Sharing among Smart Energy Buildings by Distributed Transaction," IEEE Transactions on Smart Grid, 2019.

[4] C. P. Mediwaththe, M. Shaw, S. K. Halgamuge, D. Smith, and P. M. J. Scott, "An Incentive-compatible Energy Trading Framework for Neighborhood Area Networks with Shared Energy Storage," IEEE Transactions on Sustainable Energy, 2019.

[5] S. Cui, Y.-W. Wang, C. Li, and J.-W. J. Xiao, "Prosumer Community: A Risk Aversion Energy Sharing Model," IEEE Transactions on Sustainable Energy, 2019.

[6] N. Liu, J. Wang, and L. J. Wang, "Hybrid Energy Sharing for Multiple Microgrids in an Integrated Heat-Electricity Energy System," IEEE Transactions on Sustainable Energy, 2018.

[7] A. Fleischhacker, H. Auer, G. Lettner, and A. J. Botterud, "Sharing solar PV and energy storage in apartment buildings: resource allocation and pricing," IEEE Transactions on Smart Grid, 2018.

[8] S. Senemar, A. R. Seifi, M. Rastegar, and M. J. Parvania, "Probabilistic Optimal Dynamic Planning of Onsite Solar Generation for Residential Energy Hubs," IEEE Systems Journal, 2019.

[9] X. Zhang and S. J. Grijalva, "A data-driven approach for detection and estimation of residential PV installations," IEEE Transactions on Smart Grid, vol. 7, no. 5, pp. 2477-2485, 2016.

[10] Y. Ghiassi-Farrokhfal, F. Kazhamiaka, C. Rosenberg, and S. J. Keshav, "Optimal design of solar PV farms with storage," IEEE transactions on sustainable energy, vol. 6, no. 4, pp. 1586-1593, 2015.

[11] M. Alhaider and L. J. Fan, "Planning Energy Storage and Photovoltaic Panels for Demand Response With Heating Ventilation and Air Conditioning Systems," IEEE Transactions on Industrial Informatics, vol. 14, no. 11, pp. 5029-5037, 2018.

[12] Z. Li, Y. Xu, S. Fang, and S. Mazzoni, "Optimal placement of heterogeneous distributed generators in a grid-connected multi-energy microgrid under uncertainties," IET Renewable Power Generation, vol. 13, no. 14, pp. 2623-2633, 2019. 
[13] G. Cáceres, S. Nasirov, H. Zhang, and G. J. Araya-Letelier, "Residential solar PV planning in Santiago, Chile: Incorporating the PM10 parameter," Sustainability, vol. 7, no. 1, pp. 422-440, 2015.

[14] A. Yoza, A. Yona, T. Senjyu, and T. J. Funabashi, "Optimal capacity and expansion planning methodology of PV and battery in smart house," Renewable Energy, vol. 69, pp. 25-33, 2014.

[15] M. Farivar and S. H. J. Low, "Branch flow model: Relaxations and convexification-Part I," IEEE Transactions on Power Systems, vol. 28, no. 3, pp. 2554-2564, 2013.

[16] S. Kim, M. J. Kojima, and software, "Second order cone programming relaxation of nonconvex quadratic optimization problems," Optimization methods, vol. 15, no. 3-4, pp. 201-224, 2001.

[17] M. Farivar and S. H. Low, "Branch Flow Model: Relaxations and Convexification--Part I," IEEE Trans. Power Syst., vol. 28, no. 3, pp. 2554-2564, 2013, doi: 10.1109/TPWRS.2013.2255317.

[18] J. P. Painuly, "Barriers to renewable energy penetration; a framework for analysis," Renewable Energy, vol. 24, no. 1, pp. 73-89, 2001.

[19] T. J. Foxon, R. Gross, A. Chase, J. Howes, A. Arnall, and D. Anderson, "UK innovation systems for new and renewable energy technologies: drivers, barriers and systems failures," Energy policy, vol. 33, no. 16, pp. 2123-2137, 2005.

[20] L. Byrnes, C. Brown, J. Foster, and L. D. Wagner, "Australian renewable energy policy: Barriers and challenges," Renewable Energy, vol. 60, pp. 711-721, 2013.

[21] N. Phuangpornpitak and S. Tia, "Opportunities and challenges of integrating renewable energy in smart grid system," Energy Procedia, vol. 34, pp. 282-290, 2013.

[22] "Barriers to Renewable Energy Technologies." https://www.ucsusa.org/resources/barriers-renewable-energy-technologie s.

[23] H. Heitsch and W. Römisch, "Scenario reduction algorithms in stochastic programming," Computational optimization and applications, vol. 24, no. 2-3, pp. 187-206, 2003.

[24] R. B. Myerson, Game theory. Harvard university press, 2013.

[25] N. Liu, X. Yu, C. Wang, and J. J. Wang, "Energy sharing management for microgrids with PV prosumers: A Stackelberg game approach," IEEE Transactions on Industrial Informatics, vol. 13, no. 3, pp. 1088-1098, 2017.

[26] J. Li, Z. Xu, J. Zhao, and C. Zhang, "Distributed Online Voltage Control in Active Distribution Networks Considering PV Curtailment," IEEE Transactions on Industrial Informatics, 2019.

[27] H. W. Kuhn and A. W. Tucker, "Nonlinear programming, in (J. Neyman, ed.) Proceedings of the Second Berkeley Symposium on Mathematical Statistics and Probability," ed: University of California Press, Berkeley, 1951.

[28] Z.-Q. Luo, J.-S. Pang, and D. Ralph, Mathematical programs with equilibrium constraints. Cambridge University Press, 1996.

[29] G. Dantzig, Linear programming and extensions. Princeton University Press, 2016.

[30] J. Fortuny-Amat and B. J. McCarl, "A representation and economic interpretation of a two-level programming problem," Journal of the Operational Research Society, vol. 32, no. 9, pp. 783-792, 1981.

[31] I. I. J. Cplex, "V12. 1: User's Manual for CPLEX," International Business Machines Corporation, vol. 46, no. 53, p. 157, 2009.

[32] G. J. U. h. w. g. c. OPTIMIZATION, "INC. Gurobi optimizer reference manual, 2015," 2014.

[33] S. R. Shaw, S. B. Leeb, L. K. Norford, and R. W. Cox, "Nonintrusive load monitoring and diagnostics in power systems," IEEE Transactions on Instrumentation and Measurement, vol. 57, no. 7, pp. 1445-1454, 2008.

[34] M. E. Baran and F. F. J. Wu, "Network reconfiguration in distribution systems for loss reduction and load balancing," IEEE Transactions on Power Delivery, vol. 4, no. 2, pp. 1401-1407, 1989.

[35] M. Attar, O. Homaee, H. Falaghi, and P. Siano, "A novel strategy for optimal placement of locally controlled voltage regulators in traditional distribution systems," International Journal of Electrical Power \& Energy Systems, vol. 96, pp. 11-22, 2018.

[36] R. K. Kaufmann and D. J. Vaid, "Lower electricity prices and greenhouse gas emissions due to rooftop solar: empirical results for Massachusetts," Energy Policy, vol. 93, pp. 345-352, 2016.

[37] S. Sharma, Y. Xu, A. Verma, and B. K. Panigrahi, "Time-Coordinated Multi-Energy Management of Smart Buildings under Uncertainties," IEEE Transactions on Industrial Informatics, 2019.

[38] S. F. Santos, D. Z. Fitiwi, M. Shafie-Khah, A. W. Bizuayehu, C. M. Cabrita, and J. P. Catalão, "New multistage and stochastic mathematical model for maximizing RES hosting capacity-Part I: Problem formulation," IEEE Transactions on Sustainable Energy, vol. 8, no. 1, pp. 304-319, 2016.

[39] Z. Liu, F. Wen, and G. Ledwich, "Optimal planning of electric-vehicle charging stations in distribution systems," IEEE Transactions on Power Delivery, vol. 28, no. 1, pp. 102-110, 2012.

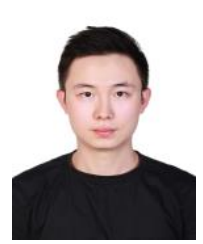

Xu Xu (S'18 M'19) received the M.E and Ph.D. degrees from The Hong Kong Polytechnic University, Hong Kong SAR in 2016 and 2019, respectively. Dr Xu is with the Department of Electrical Engineering, The Hong Kong Polytechnic University, Hung Hom, Hong Kong and also with the School of Electrical and Electronic Engineering, Nanyang Technological University, Singapore. His current research interests include power system planning and operation, renewable power integration, energy management, and artificial intelligence application in power engineering.

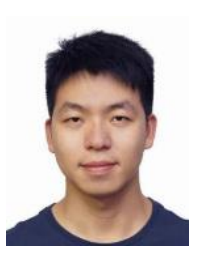

Jiayong Li (S'16-M'19) received the B.Eng. degree from Zhejiang University, Hangzhou, China, in 2014, and the Ph.D. degree from The Hong Kong Polytechnic University, Hong Kong, in 2018. He is currently an Assistant Professor with the College of Electrical and Information Engineering, Hunan University, Changsha, China. He was a Postdoctoral Research Fellow with The Hong Kong Polytechnic University and a Visiting Scholar with Argonne National Laboratory, Argonne, IL, USA. His research interests include power economics, energy management, distribution system planning and operation, renewable energy integration, and demand-side energy management.

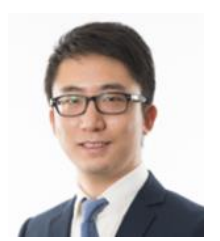

Yan Xu (S'10-M'13-SM'19) received the B.E. and M.E degrees from South China University of Technology, Guangzhou, China in 2008 and 2011, respectively, and the Ph.D. degree from The University of Newcastle, Australia, in 2013. He is now the Nanyang Assistant Professor at School of Electrical and Electronic Engineering, Nanyang Technological University (NTU), and a Cluster Director at Energy Research Institute @ NTU (ERI@N), Singapore. Previously, he held The University of Sydney Postdoctoral Fellowship in Australia. His research interests include power system stability and control, microgrid, and data-analytics for smart grid applications. Dr Xu is an Editor for IEEE Transactions on Smart Grid, IEEE Transactions on Power Systems, IEEE Power Engineering Letters, CSEE Journal of Power and Energy Systems, and an Associate Editor for IET Generation, Transmission \& Distribution.

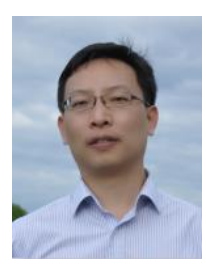

Zhao $\mathrm{Xu}$ (M'06-SM'13) received the Ph.D. degree in electrical engineering from The University of Queensland, Brisbane, Australia, in 2006.From 2006 to 2009, he was an Assistant and later Associate Professor with the Centre for Electric Technology, Technical University of Denmark, Lyngby, Denmark. Since 2010, he has been with Hong Kong Polytechnic University. His research interests include demand side, grid integration of wind power, electricity market planning and management, and $\mathrm{AI}$ applications. He is an Editor of the Electric Power Components and Systems journal.

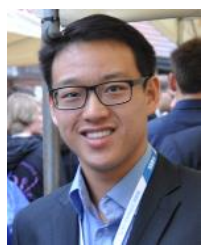

Chun Sing Lai (S'11, M'19) received the B.Eng. (First Class Honours) in electrical and electronic engineering from Brunel University London, UK and D.Phil. in engineering science from the University of Oxford, UK in 2013 and 2019, respectively. Dr Lai is currently a Lecturer at Department of Electronic and Computer Engineering, Brunel University London, UK and also a Visiting Academic with the Department of Electrical Engineering, Guangdong University of Technology, China. He is a member of Brunel Institute of Power Systems. From 2018 to 2020, he was an Engineering and Physical Sciences Research Council Research Fellow with the School of Civil Engineering, University of Leeds. He is Secretary of the IEEE Smart Cities Publications Committee and Acting EiC of IEEE Smart Cities Newsletters. He organized the workshop on Smart Grid and Smart City, IEEE SMC 2017 in Canada. Dr Lai is the Working Group Chair for IEEE P2814 Techno-economic Metrics Standard for Hybrid Energy and Storage Systems. His current interests are in power system optimization, energy system modelling, data analytics, and energy economics for low carbon energy networks and energy storage systems. 